\title{
Molecular mechanisms of the metabolite 4-hydroxytamoxifen of the anticancer drug tamoxifen: use of a model microorganism
}

\author{
J.P. Monteiro ${ }^{\text {a }}$, J.D. Martins ${ }^{\mathrm{a}}$, P.C. Luxo ${ }^{\mathrm{b}}$, A.S. Jurado ${ }^{\mathrm{a}, *}$, V.M.C. Madeira ${ }^{\mathrm{a}}$ \\ ${ }^{a}$ Centro de Neurociencias, Universidade de Coimbra, 3000 Coimbra, Portugal

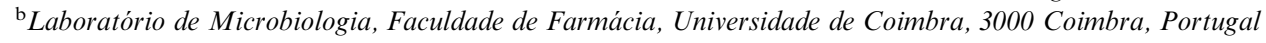

Accepted 31 May 2003

\begin{abstract}
A strain of the thermophilic eubacterium Bacillus stearothermophilus was used as a model system to identify membrane mediated cytotoxic effects of 4-hydroxytamoxifen, following previous studies with tamoxifen. With this experimental approach we attempted to further clarify tamoxifen and 4-hydroxytamoxifen membrane interactions often evoked as responsible for their multiple cellular effects. Bacterial growth and the oxygen consumption rate provided quantitative data of the cytotoxic action of hydroxytamoxifen. The effects of hydroxytamoxifen on the physical properties of bacterial lipid membrane preparations were also evaluated by fluorescence polarization of 1,6-diphenyl-1,3,5-hexatriene. Cultures of B. stearothermophilus grown in a complex medium containing hydroxytamoxifen in the concentration range of 1 to $7 \mu \mathrm{M}$ exhibited progressively longer lag adapting periods, decreased specific growth rates and lower growth yields, as compared to control cultures. Hydroxytamoxifen also affected the electron redox flow of B. stearothermophilus protoplasts and induced significant perturbation of the structural order of bacterial lipid dispersions. We concluded that the bacterial model provides useful information about the nature and repercussion of membrane physical interactions of this lipophilic drug, on the basis of an easy and economic methodology.
\end{abstract}

(C) 2003 Elsevier Ltd. All rights reserved.

Keywords: Tamoxifen; Hydroxytamoxifen; Fluorescence polarization; Bacillus stearothermophilus; Bacterial growth; Lipid membrane

\section{Introduction}

Tamoxifen (TAM) is a nonsteroidal anti-oestrogen that has been used as first line therapy in breast cancer (Fisher and Redmond, 1991; Nayfield et al., 1991) and 4-hydroxytamoxifen (OHTAM) is its more active metabolite in humans (Kemp et al., 1983). Although the mechanisms by which TAM exerts its antiproliferative activity are not yet clarified, evidence has accumulated to indicate that TAM can influence cell proliferation by means not restricted to the classical oestrogen receptor system (Kon, 1989). Multiple cellular effects of TAM and OHTAM have been reported (Wiseman et al., 1990;

Abbreviations: DPH, 1,6-diphenyl-1,3,5-hexatriene; DPH-PA, 3(p-(6-phenyl)-1,3,5-hexatrienyl)phenylpropionic acid; NADH, nicotinamide adenine dinucleotide, reduced form; OHTAM, 4-hydroxytamoxifen; TAM, tamoxifen; TMPD, N,N, $\mathrm{N}^{\prime}, \mathrm{N}^{\prime}$-tetramethyl- $p$ phenylenediamine.

* Corresponding author. Tel.: + 351-239-834729; fax: + 351-239826798.

E-mail address: asjurajo@ci.uc.pt (A.S. Jurado).
Custódio et al., 1994, 1996 and 1998) and the cytotoxic effects are consistently related with multiple interactions at the level of the cell membrane (Custódio et al., 1993; Luxo et al., 1996, 1998, 2000). Different locations of TAM and OHTAM across the bilayer thickness, inferred by the perturbing effects on the structural order at different depths within the membrane (Custódio et al., 1993), have been suggested to contribute to specific TAM and OHTAM interactions with receptors, regulatory proteins and enzymes distributed in different lipid domains. These interactions may be involved in multiple action mechanisms of this anti-oestrogenic drug leading to inhibition of cell proliferation. To elucidate the molecular mechanisms of TAM at the membrane level and its antiproliferative effects not related to oestrogen receptor binding, a microorganism grown under laboratory-controlled conditions has been used as a model system (Luxo et al., 1996, 1998, 2000). The present work follows a sequence of previous studies of the effects of TAM on growth and membrane lipid composition of B. stearothermophilus (Luxo et al., 1996, 
1998). This microorganism has been selected owing to the strict dependence of growth on the physical state of membrane lipids as affected by temperature, drugs and divalent cations (Jurado et al., 1987; Luxo et al., 1996; Donato et al., 1997a,b; Rosa et al., 2000a) and its ability to adapt to alterations of the environmental conditions, by changing membrane lipid composition (Jurado et al., 1991; Luxo et al., 1998; Donato et al., 2000; Rosa et al., 2000b). To further clarify the molecular mechanisms of action of lipophilic drugs, particularly the influence of membrane biophysical alterations on toxicity effects, we extended the studies to 4-hydroxytamoxifen, attempting to establish a correlation between the effects of this compound on growth, respiratory activity and membrane lipid organization.

\section{Materials and methods}

\subsection{Chemicals}

Tamoxifen (TAM) and 1,6-diphenyl-1,3,5-hexatriene (DPH) were obtained from Sigma Chemical co. 4Hydroxytamoxifen (OHTAM) was obtained from Amersham.

\subsection{Cultures}

The strain of Bacillus stearothermophilus and the conditions for its maintenance and growth have been described previously (Jurado et al., 1987). Liquid cultures were started with an early stationary inoculum and were grown in one liter Erlenmeyer flasks containing $200 \mathrm{ml}$ of diluted L-Broth, shaken at $100 \mathrm{rpm}$ in a New Brunswick water bath shaker. TAM and OHTAM from concentrated ethanolic solutions were added to the growth medium to obtain concentrations ranging from 1 to $7 \mu \mathrm{M}$. The bacterial growth in media containing TAM or OHTAM was performed in silanized Erlenmeyer flasks, since this drug strongly binds to glass material (Custódio et al., 1991). Growth was measured by turbidimetry at $610 \mathrm{~nm}$ in a Bausch \& Lomb Spectronic 21 spectrophotometer.

\subsection{Lipid analysis}

Cells, grown up to the beginning of the stationary phase, were harvested by low-speed centrifugation and washed three times with buffer (10 mm Tris-Cl, $\mathrm{pH} 7.0)$. The lipids were extracted by the method of Bligh and Dyer (1959) and quantified by measuring the amount of inorganic phosphate (Bartlett, 1959) after hydrolysis of the extracts at $180{ }^{\circ} \mathrm{C}$ in $70 \% \mathrm{HClO}_{4}$ (Bottcher et al., 1961). The polar lipids were isolated by preparative thin layer chromatography as previously described (Jurado et al., 1991)

\subsection{Liposomes}

Aliquots from lipid solutions in $\mathrm{CHCl}_{3}$ (polar lipid extract) containing $2.325 \mathrm{mg}$ of lipid were evaporated to dryness on a rotary evaporator. The dry residues were hydrated under $\mathrm{N}_{2}$ atmosphere at $55^{\circ} \mathrm{C}$ by gentle shaking with $5 \mathrm{ml}$ of $50 \mathrm{~mm} \mathrm{KCl}$ and $10 \mathrm{~mm}$ Tris-maleate $(\mathrm{pH} 7.0)$ and multilamellar vesicles were obtained. Then, the suspensions were vortexed for $1 \mathrm{~min}$ to disperse aggregates.

\subsection{Incorporation of probes and OHTAM into liposomes of bacterial polar lipids}

The fluidity probe 1,6-diphenyl-1,3,5-hexatriene (DPH) in dimethylformamide was injected (few $\mu$ l) into liposomes suspensions (345 $\mu \mathrm{M}$ in phospholipid), as described previously (Antunes-Madeira et al., 1994) to give a lipid/probe molar ratio of 200. After incubation at $55{ }^{\circ} \mathrm{C}$ in the dark, for $18-20 \mathrm{~h}$, OHTAM was added from an ethanolic solution and the mixtures were allowed to equilibrate for $20 \mathrm{~min}$ at $37{ }^{\circ} \mathrm{C}$, before fluorescence measurements.

\subsection{Fluorescence polarization measurements}

The fluorimetric measurements were performed with a Perkin-Elmer spectrofluorimeter, model MPF-66, with a thermostated cell holder. The excitation was set at 336 $\mathrm{nm}$ and the emission at $450 \mathrm{~nm}(5 \mathrm{~nm}$ excitation and 6 $\mathrm{nm}$ band pass).

All fluorescence measurements were corrected for the contribution of light scattering by using appropriate blanks without added probes. The degree of fluorescence polarization (P) was calculated according Shinitzky and Barenholz (1978) from the equation:

$\mathrm{P}=\frac{\mathrm{I}_{\|}-\mathrm{I}_{\perp} \cdot \mathrm{G}}{\mathrm{I}_{\|}+\mathrm{I}_{\perp} \cdot \mathrm{G}}$

where $I_{\|}$and $I_{\perp}$ are the intensities of the light emitted with its polarization plane parallel and perpendicular to that of exciting beam. $G$ is the correction factor for instrument polarization, given by the ratio of vertically to the horizontally polarized emission components when the excitation light is polarized in the horizontal direction.

\subsection{Statistic analysis of data}

The results of specific growth rates and final cell densities are presented as means \pm SEM of the number of experiments indicated. Multiple comparisons were performed using one-way ANOVA, with the StudentNewman-Keuls as a post-test. $P<0.05$ was considered significant. 


\section{Results}

\subsection{Effect of hydroxytamoxifen on the growth of B. stearothermophilus}

The strain of B. stearothermophilus was grown at $65{ }^{\circ} \mathrm{C}$ (in the optimal temperature range) in a complex medium (diluted L-Broth) to which tamoxifen (TAM) and hydroxytamoxifen (OHTAM) from concentrated ethanolic solutions were added, to obtain concentrations ranging from 1 to $7 \mu \mathrm{M}$; control cultures were

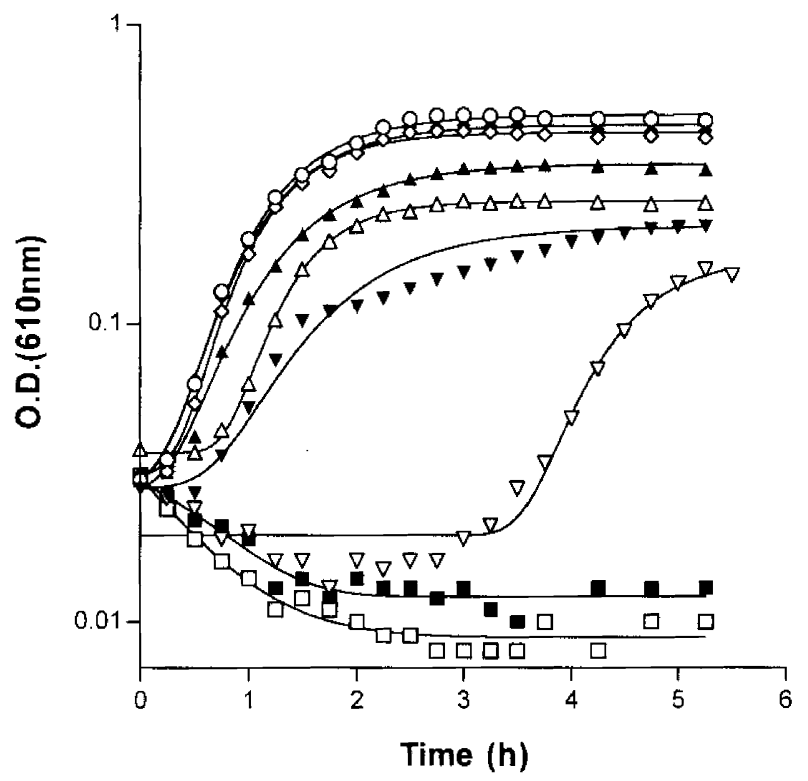

Fig. 1. Effect of tamoxifen (TAM) and hydroxytamoxifen (OHTAM) on the growth of cultures of B. stearothermophilus, at $65^{\circ} \mathrm{C}$. Cells were grown in a basal medium (diluted L-Broth) without drugs $(\bigcirc)$ and with $1 \mu \mathrm{M}(\diamond), 3 \mu \mathrm{M}(\triangle), 5 \mu \mathrm{M}(\nabla)$ and $7 \mu \mathrm{M}(\square)$ TAM or $1 \mu \mathrm{M}(\diamond), 3$ $\mu \mathrm{M}(\boldsymbol{\Delta}), 5 \mu \mathrm{M}(\boldsymbol{\nabla})$ and $7 \mu \mathrm{M}(\boldsymbol{\square})$ OHTAM. The results are typical of three separate experiments. grown in a medium without drugs, but with $0.0375 \%(\mathrm{v} /$ v) ethanol (i.e. the maximum amount of solvent used).

According to previous studies (Luxo et al., 1996), the addition of TAM inhibited growth as a function of concentration (Fig. 1). Under the same experimental conditions, using the same inoculum and in the same concentration range $(1-7 \mu \mathrm{M})$, it was shown that OHTAM inhibited growth too, although its effect on the bacterial yield was significantly lower as compared with TAM. Growth inhibition with increasing drug concentrations was characterized by progressively longer lag periods, lower specific growth rates and lower final cell densities, as documented in Fig. 1 and Table 1. The lag times in Table 1 correspond to the experiment of Fig. 1. Since the variability of this growth parameter in different growth experiments is appreciable (owing to several conditions, viz. the age of the inoculum), means were not determined. With high drugs concentrations (5 $\mu \mathrm{M}$ ), TAM induced a much longer lag time (about $3 \mathrm{~h}$ and $45 \mathrm{~min}$ ) than OHTAM, and, with both drugs, a significant growth rate is followed by an abrupt cessation of growth with bacterial yields significantly depressed.

\subsection{Physical effects of hydroxytamoxifen on bilayers of bacterial polar lipids}

Fluidity is a physical membrane parameter very susceptible to perturbations by membrane active drugs (Antunes-Madeira et al., 1994; Balasubramanian and Straubinger, 1994; Videira et al., 1999; Luxo et al., 2000). Therefore, we studied the effects of OHTAM on the fluidity of bacterial polar lipid dispersions by fluorescence polarization of 1,6-diphenyl-1,3,5-hexatriene (DPH). This physical parameter is related with probe rotational diffusion strongly dependent on fluidity (Shinitzky and Barenholz, 1978). DPH, a probe buried in the bilayer core (Shinitzky and Barenholz, 1978),

Table 1

Lag times, specific growth rates and cell densities (O.D.) reached in the stationary phase of cultures of Bacillus stearothermophilus grown in media with different concentrations of TAM or OHTAM

\begin{tabular}{llll}
\hline $\begin{array}{l}\text { Additives to the growth } \\
\text { medium }\end{array}$ & $\begin{array}{l}\text { Lag time } \\
(\mathrm{h})\end{array}$ & $\begin{array}{l}\text { Specific growth rate } \\
\left(\mathrm{h}^{-1}\right)^{\mathrm{a}}\end{array}$ & $\begin{array}{l}\text { Final cell densities } \\
(\% \text { of control })^{\mathrm{a}}\end{array}$ \\
\hline None (control) & 0.466 & $2.359 \pm 0.035(8)$ & $100(4)$ \\
$1 \mu \mathrm{M}$ TAM & 0.522 & $2.266 \pm 0.045(3) \mathrm{n} . \mathrm{s}$. & $87.863 \pm 0.748(3) \mathrm{n} . \mathrm{s}$. \\
$3 \mu \mathrm{M}$ TAM & 0.891 & $1.681 \pm 0.083(3)^{* * *}$ & $53.503 \pm 3.670(3)^{* * *}$ \\
$5 \mu \mathrm{M}$ TAM & 3.705 & $1.305 \pm 0.136(4)^{* * *, \diamond}$ & $33.170 \pm 0.609(4)^{* * *, \diamond}$ \\
$1 \mu \mathrm{M}$ OHTAM & 0.446 & $2.189 \pm 0.102(3) \mathrm{n} . \mathrm{s}$. & $94.167 \pm 2.351(3) \mathrm{n} . \mathrm{s}$. \\
$3 \mu \mathrm{M}$ OHTAM & 0.495 & $1.770 \pm 0.063(4)^{* * *}$ & $78.927 \pm 6.810(4) \mathrm{n} . \mathrm{s}^{*}$ \\
$5 \mu \mathrm{M}$ OHTAM & 0.850 & $1.473 \pm 0.062(4)^{* * * \diamond}$ & $59.355 \pm 10.539(4)^{* * *} \diamond, \star *$ \\
\hline
\end{tabular}

n.s. not significant, ${ }^{* * *} P<0.001$; cultures grown with $5 \mu \mathrm{M}$ TAM vs. cultures grown with $3 \mu \mathrm{M}$ TAM or cultures grown with $5 \mu \mathrm{M}$ OHTAM vs. cultures grown with $3 \mu \mathrm{M}$ OHTAM $(\diamond P<0.05 ; \diamond \diamond P<0.01)$ and cultures grown with $3 \mu \mathrm{M}$ OHTAM vs. cultures grown with $3 \mu \mathrm{M}$ TAM, or cultures grown with $5 \mu$ M OHTAM vs. cultures grown with $5 \mu$ M TAM $\left({ }^{\star} P<0.05 ;{ }^{\star \star} P<0.01\right)$.

a Values of specific growth rates and final cell densities are means \pm standard error of mean from three or more independent experiments (number in brackets) and comparisons were performed using one-way ANOVA, with the Student-Newman-Keuls as a post-test, for the following paired observations: control cultures (grown without additives) vs. cultures grown with TAM (1, 3 or $5 \mu \mathrm{M})$ or vs. cultures grown with OHTAM (1, 3 or $5 \mu \mathrm{M})$. 
provides information on this hydrophobic region of the membrane.

Fig. 2 shows the thermograms of fluorescence polarization of DPH in liposomes prepared with the polar lipid fraction of the membrane of cells grown in the control medium, at $65^{\circ} \mathrm{C}$. The addition of OHTAM (50 and $100 \mu \mathrm{m}$ ) induced a decrease of fluorescence polarization in the gel phase and along the phase transition temperature range, as a function of concentration. A moderate broadening of the transition profile was also detected. These data indicate a general induced disorder in the hydrophobic core of the lipid bilayer where DPH distributes. OHTAM effects on the lipid organization above the phase transition temperature become less evident.

\subsection{Effect of hydroxytamoxifen on oxygen consumption of B. stearothermophilus protoplasts}

Oxygen consumption was measured with a Clark oxygen electrode, using protoplasts prepared from cultures harvested in the middle of the exponential growth phase. Two substrates were chosen, NADH allowing to study the full respiratory path and ascorbate-TMPD to monitor the activity of the final oxidase segment.

When NADH was used as respiratory substrate (Fig. 3A), a significant inhibition of the oxygen consumption rate was induced by hydroxytamoxifen as a function of concentration, up to $300 \mathrm{nmol}$ per $\mathrm{mg}$ of protein. At the maximum concentration assayed, the respiratory rate was depressed to about $37 \%$ of the control. However, when ascorbate-TMPD was used as substrate (Fig. 3B), the oxygen consumption rate was insensitive to OHTAM, in the same concentration range. As expected, the oxygen consumption supported by NADH or ascorbate-TMPD was completely

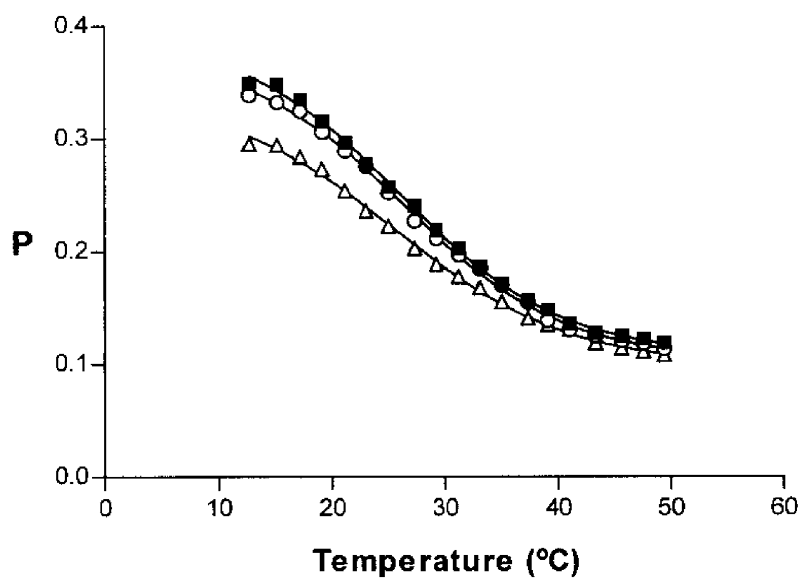

Fig. 2. Thermograms of fluorescence polarization (P) of DPH in liposomes prepared with the polar lipids of cells of B. stearothermophilus grown in the basal medium, at $65^{\circ} \mathrm{C}$. Liposomes were incubated without $(\boldsymbol{\square})$ or with $50 \mu \mathrm{M}(\bigcirc)$ and $100 \mu \mathrm{M}(\triangle)$ hydroxytamoxifen. impaired by the addition of $\mathrm{KCN}(1 \mathrm{~mm})$, as a consequence of full inhibition of cytochrome oxidase. Therefore, OHTAM interacts with the respiratory path of B. stearothermophilus at level(s) preceding the cytochrome oxidase segment (complex I and/or complex III).

\section{Discussion}

The aim of the present work, following previous studies with tamoxifen (Luxo et al., 1996, 1998 and 2000), is to elucidate membrane interactions of 4hydroxytamoxifen, attempting to establish a relationship between drug antiproliferative effects, perturbation of lipid organization and membrane dependent functions.

A strain of B. stearothermophilus has been used as a model to study the interaction of several lipophilic drugs (Luxo et al., 1996, 1998 and 2000; Rosa et al., 2000a,b) and pollutants (Donato et al., 1997a,b,c) and a remarkable parallelism between cytotoxic effects on bacterial cells and on model systems from eukaryotic cells (e.g. mithocondria) has been established (1997b).

The effects of OHTAM on growth of B. stearothermophilus at $65{ }^{\circ} \mathrm{C}$ (i.e. optimal temperature) in a complex medium (diluted L-Broth) were studied in the
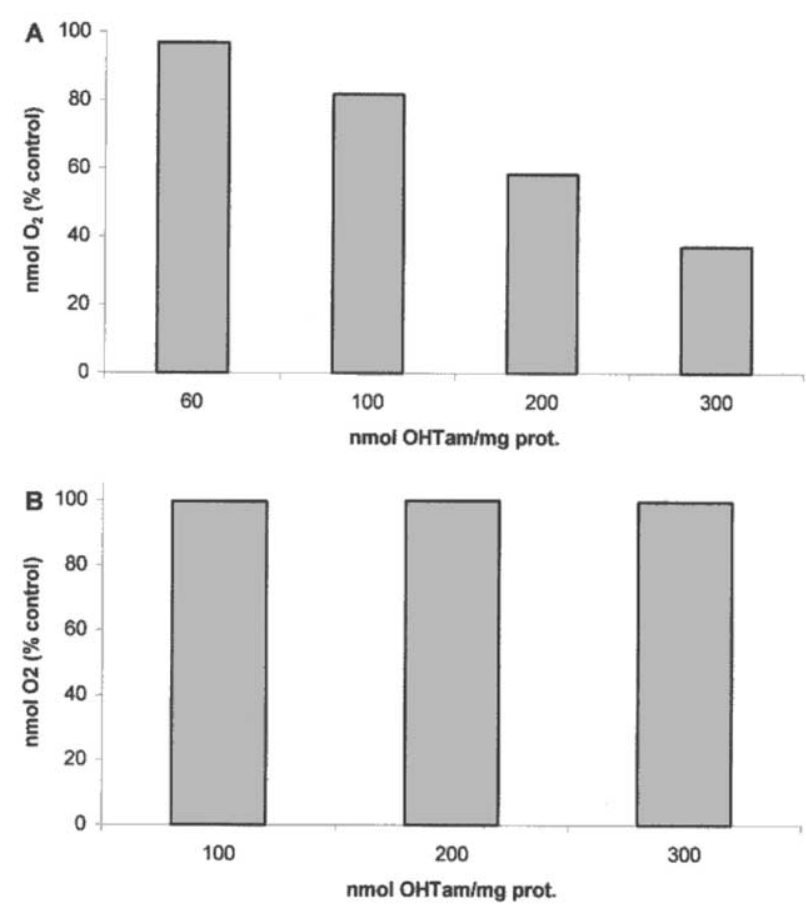

Fig. 3. Effect of hydroxytamoxifen on the oxygen consumption rate of B. stearothermophilus protoplasts when $10 \mathrm{~mm}$ NADH (A) or $10 \mathrm{~mm}$ ascorbate-600 $\mu \mathrm{M}$ TMPD (B) was used as the substrate. B. stearothermophilus was grown at $65^{\circ} \mathrm{C}$ in basal medium under control conditions (without xenobiotic compounds). The graphs show the levels of the respiratory rate as percentage of the control (protoplasts incubated for $4 \mathrm{~min}$ without xenobiotic compounds, in the presence of few microliters of dimethyl sulfoxide, the solvent). 
concentration range up to $7 \mu \mathrm{M}$. The effects were compared with effects already reported for TAM (Luxo et al., 1996). It was shown that bacterial growth is impaired as OHTAM concentration increases, and $7 \mu \mathrm{M}$ OHTAM or TAM induced a complete cessation of growth. In the same experiment, the bacterial yield is consistently more affected by TAM than by OHTAM, at the same concentrations. On the other hand, $5 \mu \mathrm{M}$ TAM, at variance with OHTAM, induced a very long lag phase. Previous studies (Luxo et al., 1998) showed that $B$. stearothermophilus controls the membrane lipid composition in response to tamoxifen, to compensate for perturbations of lipid packing.

The effects of OHTAM on the fluidity of lipid bilayers composed of bacterial polar lipids, including several phospholipid classes (phosphatidylethanolamine, phosphatidylglycerol, cardiolipin and a phosphoglycolipid) and species (Jurado et al., 1991) were investigated using fluorescence polarization of DPH. Since probe rotation is mainly restricted by the molecular packing of the lipids, a high polarization value, reflecting a limited rotational diffusion of DPH, reports a high structural order or a low membrane fluidity in the hydrophobic core of the membrane, where DPH is preferentially located. As illustrated in Fig. 2, OHTAM disorders the lipid packing, as reflected by a decrease of fluorescence polarization of DPH in the gel phase and along the phase transition temperature range, in accordance with model studies using single phospholipid liposomes (Custódio et al., 1993). In the fluid phase, no significant effects were noticed on lipid organization, in agreement with studies performed with fluid sarcoplasmic reticulum (SR) native membranes or SR lipid dispersions (Custódio et al., 1993).

A main membrane function strictly dependent on the physical properties of the lipid bilayer is the respiratory activity, here followed in protoplasts reacted with different OHTAM concentrations. The oxygen consumption elicited by NADH, monitored polarographically with a Clark oxygen electrode, is strongly inhibited by OHTAM. Since there are no effects on the oxygen consumption when the final oxidase segment was assayed (using ascorbate-TMPD as substrate), in the OHTAM concentration range from 100 to $300 \mathrm{nmol} / \mathrm{mg}$ protein, it is concluded that OHTAM interacts with the respiratory path of $B$. stearothermophilus at level(s) preceding the cytochrome oxidase segment, in agreement with previous studies with TAM (Luxo et al., 2001). Assays of the activities of the cytochrome oxidase, succinatecytochrome $\mathrm{c}$ reductase and $\mathrm{F}_{1}$ ATPase (reverse of $\mathrm{F}_{\mathrm{o}} \mathrm{F}_{1}$ activity) are currently under study.

In conclusion, OHTAM inhibits bacterial growth, perturbs membrane lipid organization and impairs the respiratory activity of $B$. stearothermophilus, suggesting a possible relationship between membrane interactions of this lipophilic drug and its antiproliferative effects.
Although we are aware of the limitations of the meaning of these results in a therapeutic perspective, our bacterial model is, however, an experimental approach to seek basic aspects of the biochemical activity of TAM and its metabolite OHTAM, namely: (i) growth-inhibiting activity of tumor cells not involving the oestrogen receptor binding model (Kon, 1989) and (ii) the plethora of cellular effects promoted by TAM and OHTAM most of which not yet fully understood. There is growing evidence that the multiple and distinct biological effects of these lipophilic compounds involve specific partitioning in different lipid domains (Custódio et al., 1993). This phenomenon can not be identified in mammalian cells owing to the complex membrane structure, but membrane models (e.g. liposomes) or bacterial membranes provide useful approaching data. The bacterial model offers over eukaryotic cells the following advantages: (i) bacterial growth in laboratory controlled conditions is easy and economic; (ii) the model avoids ethical issues associated with animal use and is an alternative in vitro toxicity test; (iii) the model allows to correlate, in the same system, the toxicity of the xenobiotic as assessed in vivo by growth disturbances and in vitro toxicity effects on membrane dependent cell functions and physical perturbations of membrane physical properties. Therefore, these experimental data, combined with other data collected from similar studies using different model systems, may provide useful information for a better understanding of the molecular mechanisms of action of TAM and OHTAM, likely involved either in their therapeutic activity or in cytotoxic side effects. Additionally, the study of the lipid composition changes undergone by bacterial cells to compensate for chemical stress (Luxo et al., 1998) may also contribute to elucidate the mechanisms underlying the resistance of tumor cells to chemotherapy and to establish clues to enhance neoplastic sensitivity to cytostatic drugs and to implement new anticancer strategies.

\section{References}

Antunes-Madeira, M.C., Videira, R.A., Madeira, V.M.C., 1994 Effects of parathion on membrane organization and its implications for the mechanisms of toxicity. Biochimica et Biophysica Acta 1190, 149-154.

Balasubramanian, S.V., Straubinger, R.M., 1994. Taxol lipid interactions: Taxol-dependent effects on the physical properties of model membranes. Biochemistry 33, 8941-8947.

Bartlett, G.R., 1959. Phosphorus assay in column chromatography. Journal of Biological Chemistry 234, 466-468.

Bligh, E.G., Dyer, W.J., 1959. A rapid method of total lipid extraction and purification. Canadian Journal of Biochemistry and Physiology 37, 911-917.

Böttcher, C.J.F., van Gent, C.M., Pries, C., 1961. A rapid and sensitive submicro phosphorus determination. Analytica Chimica Acta 24, 203-204. 
Custódio, J.B.A., Almeida, L.M., Madeira, V.M.C., 1991. A reliable and rapid procedure to estimate drug partitioning in biomembranes. Biochemical and Biophysical Research Communications 176, 10791085.

Custódio, J.B.A., Almeida, L.M., Madeira, V.M.C., 1993. The active metabolite hydroxytamoxifen of the anticancer drug tamoxifen induces structural changes in membranes. Biochimica et Biophysica Acta 1153, 308-314.

Custódio, J.B.A., Dinis, T.C.P., Almeida, L.M., 1994. Tamoxifen and hydroxytamoxifen as intramembraneous inhibitors of lipid peroxidation. Evidence for peroxyl radical scavenging activity. Biochemical Pharmacology 47, 1989-1998.

Custódio, J.B.A., Almeida, L.M., Madeira, V.M.C., 1996. The effect of the anticancer drugs tamoxifen and hydroxytamoxifen on the calcium pump of isolated sarcoplasmic reticulum vesicles. Toxicology in Vitro 10, 523-531.

Custódio, J.B.A., Moreno, A.J.M., Wallace, K.B., 1998. Tamoxifen inhibits induction of the mitochondrial permeability transition by $\mathrm{Ca}^{2+}$ and inorganic phosphate. Toxicology and Applied Pharmacology 152, 10-17.

Donato, M.M., Jurado, A.S., Antunes-Madeira, M.C., Madeira, V.M.C., 1997a. Bacillus stearothermophilus as a model to evaluate membrane toxicity of a lipophilic environmental pollutant (DDT). Archives of Environmental Contamination and Toxicology 33, 109-116.

Donato, M.M., Jurado, A.S., Antunes-Madeira, M.C., Madeira, V.M.C., 1997b. Comparative study of the toxic actions of DDT and DDE on the growth and respiratory activity of a microorganism used as a model. Applied Environmetal Microbiology 63, 49484951.

Donato, M.M., Jurado, A.S., Antunes-Madeira, M.C., Madeira, V.M.C., 1997c. Effects of a lipophilic environmental pollutant (DDT) on the phospholipid and fatty acid contents of Bacillus stearothermophilus. Archives of Environmental Contamination and Toxicology 33, 341-349.

Donato, M.M., Jurado, A.S., Antunes-Madeira, M.C., Madeira, V.M.C., 2000. Membrane lipid composition of Bacillus stearothermophilus as affected by lipophilic environmental pollutants: an approach to membrane toxicity assessment. Archives of Environmental Contamination and Toxicology 39, 145-153.

Fisher, B., Redmond, C., 1991. New perspective on cancer of the collateral breast; a marker for assessing tamoxifen as a preventive agent. Journal of the National Cancer Institute 18, 1278-1280.

Jurado, A.S., Santana, A.C., Costa, M.S., Madeira, V.M.C., 1987. Influence of divalent cations on the growth and morphology of
Bacillus stearothermophilus. Journal of General Microbiology 133, 507-513.

Jurado, A.S., Pinheiro, T.J.T., Madeira, V.M.C., 1991. Physical studies on membrane-lipids of Bacillus stearothermophilus. Temperature and calcium effects. Archives of Biochemistry and Biophysics 289, 167-179.

Kemp, J.V., Adam, H.K., Wakeling, A.E., Slater, R., 1983. Identification and biological activity of tamoxifen metabolites in human serum. Biochemical Pharmacology 32, 2045-2052.

Kon, O.L., 1989. Estrogens, antiestrogens and cell proliferation. BioEssays 10, 210-214.

Luxo, C., Jurado, A.S., Custódio, J.B.A., Madeira, V.M.C., 1996. Use of Bacillus stearothermophilus as a model to study tamoxifen membrane interactions. Toxicology in Vitro 10, 463-471.

Luxo, C., Jurado, A.S., Madeira, V.M.C., 1998. Lipid composition changes induced by tamoxifen in a bacterial model system. Biochimica et Biophysica Acta 1369, 71-84.

Luxo, C., Jurado, A.S., Madeira, V.M.C., 2000. Toxicity assessment of tamoxifen by means of a bacterial model. Applied Biochemistry and Biotechnology 87, 219-232.

Luxo, C., Jurado, A.S., Custódio, J.B.A., Madeira, V.M.C., 2001. Toxic effects of tamoxifen on the growth and respiratory activity of Bacillus stearothermophilus. Toxicology in Vitro 15, 303-305.

Nayfield, S.G., Karp, J.E., Ford, L.G., Dorr, F.A., Kramer, B.S., 1991. Potential role of tamoxifen in prevention of breast cancer. Journal of the National Cancer Institute 20, 1450-1459.

Rosa, S.M.L.J., Antunes-Madeira, M.C., Jurado, A.S., Madeira, V.M.C., 2000a. Amiodarone interactions with membrane lipids and growth of Bacillus stearothermophilus used as a model. Applied Biochemistry and Biotechnology 87, 165-175.

Rosa, S.M.L.J., Antunes-Madeira, M.C., Matos, M.J., Jurado, A.S., Madeira, V.M.C., 2000b. Lipid composition and dynamics of cell membranes of Bacillus stearothermophilus adapted to amiodarone. Biochimica et Biophysica Acta 1487, 286-295.

Shinitzky, M., Barenholz, Y., 1978. Fluidity parameters of lipid regions determined by fluorescence polarization. Biochimica et Biophysica Acta 515, 367-394.

Videira, R.A., Antunes-Madeira, M.C., Madeira, V.M.C., 1999. Perturbations induced by $\alpha$ - and $\beta$-endosulfan in lipid membranes: a DSC and fluorescence polarization study. Biochimica et Biophysica Acta $1419,151-163$.

Wiseman, H., Cannon, M., Arnstein, H.R.V., 1990. Mechanism of inhibition of lipid-peroxidation by tamoxifen and 4-hydroxytamoxifen introduced in lipossomes. Similarity to cholesterol and ergosterol. FEBS Letters 274, 107-110. 